\title{
Combining scanning tunneling microscope (STM) imaging and local manipulation to probe the high dose oxidation structure of the $\operatorname{Si}(111)-7 \times 7$ surface
}

\author{
Dogan Kaya ${ }^{1,2}$, Richard J. Cobley ${ }^{3}(\bowtie)$, and Richard E. Palmer ${ }^{3}$ \\ ${ }^{1}$ Department of Electronics and Automation, Vocational School of Adana, Cukurova University, 01160 Cukurova, Adana, Turkey \\ ${ }^{2}$ Nanoscale Physics Research Laboratory, School of Physics and Astronomy, University of Birmingham, Edgbaston, Birmingham, B15 2TT, UK \\ ${ }^{3}$ College of Engineering, Swansea University, Bay Campus, Fabian Way, Swansea, SA1 8EN, UK
}

(C) The Authors 2019

Received: 15 August 2019 / Revised: 4 November 2019 / Accepted: 28 November 2019

\begin{abstract}
Understanding the atomistic formation of oxide layers on semiconductors is important for thin film fabrication, scaling down conventional devices and for the integration of emerging research materials. Here, the initial oxidation of $\mathrm{Si}(111)$ is studied using the scanning tunneling microscope. Prior to the complete saturation of the silicon surface with oxygen, we are able to probe the atomic nature of the oxide layer formation. We establish the threshold for local manipulation of inserted oxygen sites to be $+3.8 \mathrm{~V}$. Only by combining imaging with local atomic manipulation are we able to determine whether inserted oxygen exists beneath surfacebonded oxygen sites and differentiate between sites that have one and more than one oxygen atom inserted beneath the surface. Prior to the creation of the thin oxide film we observe a flip in the manipulation rates of inserted oxygen sites consistent with more oxygen inserting beneath the silicon surface.
\end{abstract}

\section{KEYWORDS}

scanning tunneling microscopy (STM), local manipulation, Si(111), oxidation

\section{Introduction}

Silicon dioxide is the most commonly used insulator and gate dielectric in integrated circuits and is also an important material for integration with emerging materials [1-4]. The oxidation of the silicon surface creates states which modify charge transport through the silicon, with these interface effects becoming more prominent as devices are scaled down. The scanning tunneling microscope (STM) is an ideal tool to perform local nanoscale chemistry directly on individual $\mathrm{O}-\mathrm{Si}$ bonding sites. The STM can induce molecular manipulation via local injection of electrons directly into single atoms and molecules. This manipulation may induce weakening (diffusion, switching) or breaking (desorption, dissociation, and transformation) of bonds, probing the chemistry and energetics of the surface [5-20].

As well as exploring the chemistry of adsorbed oxygen, the STM can be used as an atomic-resolution imaging tool to study where oxygen molecules react with the surface. Understanding the initial oxidation of the silicon surface is important to grow thin and high quality oxide films [21-23]. Therefore, the structure and electronic properties of the $\mathrm{O}-\mathrm{Si}$ interface have been examined with methods including X-ray diffraction [24], electron energy-loss spectroscopy [25], corelevel spectroscopy [26], photoelectron spectroscopy [27], and near edge X-ray absorption fine structure spectroscopy [28]. Although the oxidation states of $\mathrm{SiO}_{2}$ on $\mathrm{Si}$ can be examined with these methods, the STM can distinguish between different oxidation states spatially and directly identify the atomic positions at which reactions take place [16-18, 29-39].

In this work we study the initial oxidation of the $\mathrm{Si}(111)$ surface as a function of oxygen exposure in ultra-high vacuum using the STM. When the oxidation of silicon is studied at high dose, the surface shows only saturated silicon adatoms and at still higher doses the formation of the disordered oxide layer [40]. There remains a dosing regime between the initial oxidation and the formation of this disordered oxide layer where several structures of inserted oxygen atoms and surface oxide are still visible at atomic resolution and can be manipulated by local injection of electrons to elucidate their nature. Previously this was done for low dose oxygen exposures of 0.05 and $0.1 \mathrm{~L}$, which using measured sticking coefficients corresponded to 0.025 monolayer (ML) and $0.005 \mathrm{ML}$ coverage, respectively [29]. Here we extend this to higher doses of 0.3 and $0.4 \mathrm{~L}$, which using typical sticking coefficients corresponds approximately to 0.015 and $0.02 \mathrm{ML}$ coverage, respectively. We observe behavior that bridges existing low dose results up to the reported high dose behavior. By combining imaging and manipulation of the initial inserted oxygen we offer clearer understanding of how these sites transform under increasing oxygen exposure.

\section{Experimental}

An RHK-1000 room temperature scanning tunneling microscope was operated in ultrahigh vacuum (UHV) with a base pressure below $7 \times 10^{-11}$ Torr. Electrochemically-etched tungsten tips 
were prepared in $2 \mathrm{M} \mathrm{NaOH}$ solution, and then outgassed by direct current annealing in vacuum. Silicon (p-type boron doped, 0.01-0.02 $\Omega \cdot \mathrm{cm}$, Goodfellow) was annealed in situ to achieve the $\mathrm{Si}(111)-7 \times 7$ reconstruction[16], before exposure to 0.3 and $0.4 \mathrm{~L}$ oxygen $(99.6 \%$ purity with typical analysis of $0.4 \%$ total hydrocarbon $(\mathrm{TCH})$ as $\mathrm{CH}_{4}<20$ volumetric part per million (vppm) and $\mathrm{H}_{2} \mathrm{O}<3$ vppm, Argo International Ltd). Passive STM imaging was performed before and after injection to monitor manipulation events, using tunneling conditions where no transformations of reacted sites were observed (constant current, $+1.5 \mathrm{~V}, 100 \mathrm{pA}$ ). Drift-tracking was enabled to minimize unwanted tip displacement due to piezo creep following charge injection. All manipulations were performed with the feedback loop enabled with increasing bias voltage at a constant tunneling current of $6 \mathrm{nA}$ for $50 \mathrm{~ms}$.

\section{Results}

In the oxidation of the clean $\mathrm{Si}(111)-7 \times 7$ surface, oxygen atoms bond with either silicon surface adatoms or the rest-atoms sat lower in the surface reconstruction. If oxygen molecules are dissociatively adsorbed by the silicon adatoms, the surface adatom dangling bonds at $\sim 0.3 \mathrm{eV}$ above and below the Fermi level $\left(E_{\mathrm{F}}\right)$ are removed, reducing the density of states for the STM to tunnel into [28, 40-44]. These "dark sites" are visible in Fig. 1(a) at $+1.0 \mathrm{~V}$ gap voltage as less bright atoms within the usual surface reconstruction. If instead one or more oxygen atoms are inserted into the back bonds between the adatoms and rest atoms, the surface adatom and its unreacted bonds are shifted $\sim 0.5 \mathrm{eV}$ higher in energy compared to the unreacted adatom dangling bond site [40]. The outward relaxation of the unreacted silicon adatom also moves it physically closer to the tip $[41,45]$. We find these "bright sites" are not visible at the same gap voltage but when it is increased to $+2.0 \mathrm{~V}$ in Fig. 1(b) the shifted surface states do become accessible to the STM and bright sites appear, indicated by circles. Figure 1(c) shows a unit cell of the $\mathrm{Si}(111)-7 \times 7$ reconstruction (white rhombus) with its faulted half $(\mathrm{FH})$ and unfaulted half $(\mathrm{UH})$ sites. Corner and center positions for the $\mathrm{FH}$ and $\mathrm{UH}$ are indicated on the image by the red and blue arrows, respectively.

While the initial stages of the oxidation of the $\mathrm{Si}(111)$ surface have been studied at atomic resolution before for low doses, we extend this investigation to 0.3 and $0.4 \mathrm{~L}$ oxygen exposure at room temperature. First we investigate the nature of the oxidation by identifying the atomic positions at which reactions take place. The number of bright and dark sites were counted and sorted by the four different possible bonding positions within the unit cell with all data normalized over a $56 \mathrm{~nm} \times$ $56 \mathrm{~nm}$ area after subtraction of surface defects from the number of dark sites; presented in Table 1 .

When increasing the oxygen dose from 0.3 to $0.4 \mathrm{~L}$ the number of dark sites increased on both the faulted and unfaulted halves. The number of bright sites decreased for almost all sites but remained the same for faulted corner sites, because of the lower number of dangling bonds. Other work has found that with low oxygen exposure of $0.05 \mathrm{~L}$, bright sites are prominent mostly on the faulted side of the unit cell [30].

Combining our data with existing work by Mayne for lower dose 0.05 and $0.1 \mathrm{~L}$ oxygen exposure [29], we present the combined effects of increasing dose on the number of bright and dark sites observed with the STM in Fig. 2. At low doses more bright sites form than dark sites, but even in the transition from 0.05 to $0.1 \mathrm{~L}$ the relative increase in dark sites is higher than bright sites. We confirm that this trend increases to higher doses and observe the region where dark sites now dominate, but bright sites, which represent inserted oxygen without any surface oxygen, are still present. Beyond this coverage it has been found that only dark sites are observed prior to the formation of the disordered oxide overlayer, which we also observed when the oxygen dose was increased further [40, 46, 47].

Combining this with existing work on O-Si oxidation, we begin to construct a model of the oxidation pathway leading to the dark and bright sites viewed with the STM, shown in Fig. 3. While it is possible to directly form dark sites by oxygen bonding to silicon adatoms without any inserted oxygen, the higher number of bright sites at low dose oxygen exposure suggests that bright sites are predominantly formed. These represent the dominant initial state with an oxygen atom inserted in one of the back bonds, as seen in Fig. 3(b). With further oxygen exposure it is possible for more oxygen atoms to insert into a second back bond maintaining the bright site, or for oxygen to bond to the silicon adatom dangling bond creating
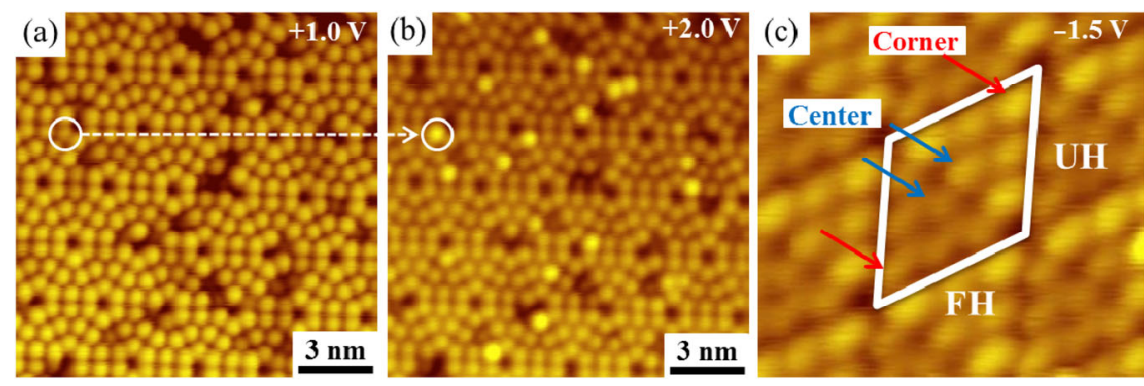

Figure 1 Room temperature STM images of the $\mathrm{Si}(111)-7 \times 7$ surface at gap voltage (a) $+1.0 \mathrm{~V}$ and (b) $+2.0 \mathrm{~V}$ after $0.3 \mathrm{~L}$ oxygen dosage, showing the appearance of bright sites at higher gap voltage. The appearance of one bright site is indicated by circles and a dashed arrow; others also appear. (c) STM image at $-1.5 \mathrm{~V} 100 \mathrm{pA}$ indicating corner positions (red arrows) and center positions (blue arrows) for the faulted half (FH) and unfaulted half (UH) of the reconstruction.

Table 1 Reaction site frequency after 0.3 and $0.4 \mathrm{~L}$ oxygen exposure to the $\mathrm{Si}(111)-7 \times 7$ surface at room temperature. The numbers of bright and dark sites were counted and normalized for a $56 \mathrm{~nm} \times 56 \mathrm{~nm}$ surface after subtracting the surface defects from the dark sites

\begin{tabular}{|c|c|c|c|c|c|c|c|c|}
\hline \multirow[b]{3}{*}{$\mathrm{O}_{2}$ dose $(\mathrm{L})$} & \multicolumn{4}{|c|}{ Bright Site } & \multicolumn{4}{|c|}{ Dark Site } \\
\hline & \multicolumn{2}{|c|}{ Faulted } & \multicolumn{2}{|c|}{ Unfaulted } & \multicolumn{2}{|c|}{ Faulted } & \multicolumn{2}{|c|}{ Unfaulted } \\
\hline & Corner & Center & Corner & Center & Corner & Center & Corner & Center \\
\hline $0.3 \mathrm{~L}$ & $18.7 \%$ & $9.2 \%$ & $7.2 \%$ & $6.9 \%$ & $20.8 \%$ & $15.2 \%$ & $14.1 \%$ & $7.9 \%$ \\
\hline $0.4 \mathrm{~L}$ & $16.8 \%$ & $7.3 \%$ & $5.4 \%$ & $3.9 \%$ & $20.6 \%$ & $26.0 \%$ & $7.6 \%$ & $12.4 \%$ \\
\hline
\end{tabular}




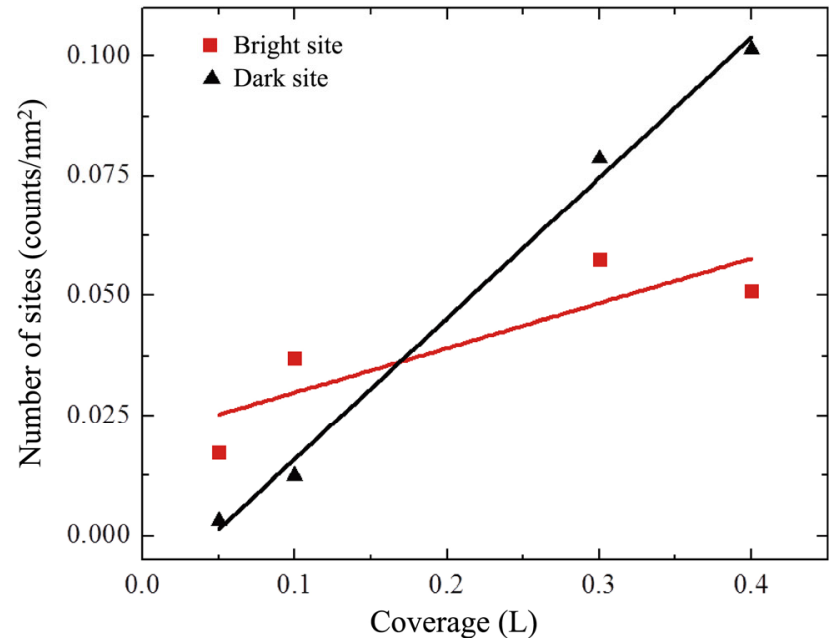

Figure 2 Initial oxidation of clean $\mathrm{Si}(111)-7 \times 7$ surface for dark and bright sites at room temperature. 0.05 and $0.1 \mathrm{~L}$ data are from Ref. [29]; 0.3 and $0.4 \mathrm{~L}$ are this work. Red squares and black triangles are for bright and dark sites, respectively. Sites are identified under passive scan conditions in the STM of $+1.5 \mathrm{~V}, 100 \mathrm{pA}$ (this work) and $+1.5 \mathrm{~V}, 200 \mathrm{pA}$ (Ref. [29]). Straight line fits to the data are to guide the eye. (a)

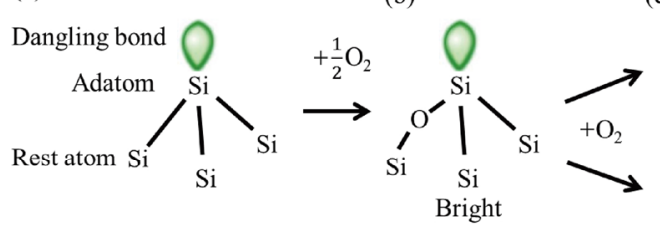

(c)
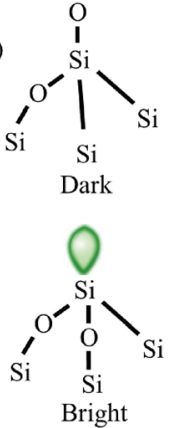

Figure 3 Model of the oxidation pathway showing the position of the oxygen atoms within the $\mathrm{Si}(111)$ surface reconstruction.

a dark site-Fig. 3(c).

This behavior can be explored further by using the STM to inject electrons directly into the silicon adatom and determining whether the associated oxygen desorbs, dissociates or transforms. Inserted oxygen is known to be more difficult to manipulate, so we first determine the voltage threshold required for bright site manipulation by measuring the number of injection sites which undergo any such reaction as a function of gap voltage, shown in Fig. 4. We determine the threshold to be $+3.8 \mathrm{~V}$, where the manipulation occurs because of electronic excitation of a surface state associated with the inserted oxygen. Although increasing the bias voltage increases the tip-sample distance in constant current mode, the electric field still increases [48], which leads to the observed increased manipulation above the voltage threshold [8].

Three examples of local site manipulations for dark sites are presented in Fig. 5. In Figs. 5(a) and 5(b) the dark site is displaced to a neighboring adatom site, indicated with a white arrow. In this manipulation the surface oxygen is transferred from the original adatom to the neighbor, resulting in a displaced dark site. The original adatom would almost certainly not have any inserted oxygen in the back bonds, otherwise with the surface oxygen removed, the original atom would become bright. In Figs. 5(c) and 5(d) the dark site transforms into a bright site. This represents either the removal of the surface oxygen revealing a bright site which already had inserted oxygen, or the manipulation of the surface oxygen into a back bond. In the

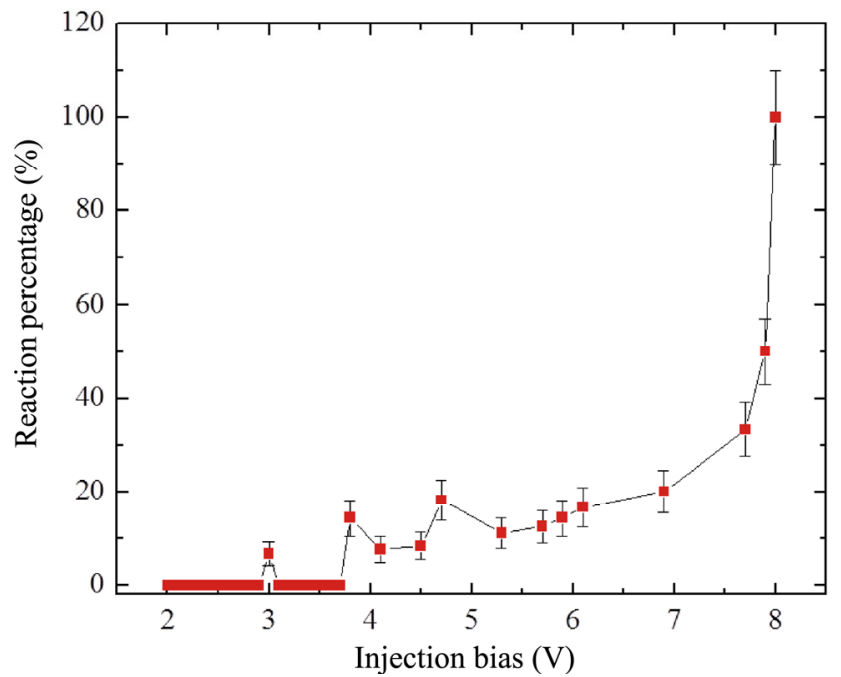

Figure 4 Reaction probability in local manipulation of the bright oxygen site as a function of injection bias applied from +2 to $+8 \mathrm{~V}$. All injections performed at fixed tunneling current of $6 \mathrm{nA}$ for $50 \mathrm{~ms}$.
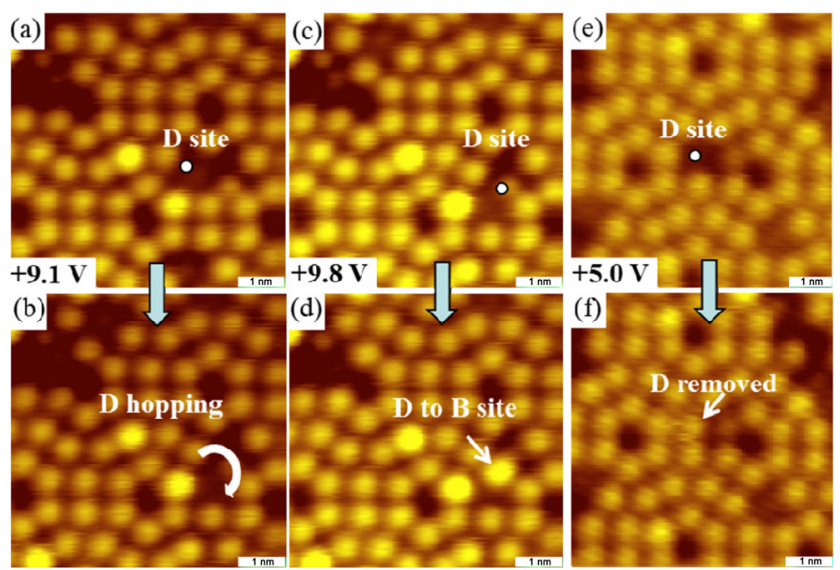

Figure 5 Manipulation of dark sites: before and after $6 \mathrm{~nm} \times 6 \mathrm{~nm}$ STM images taken at the stable imaging bias $(+1.5 \mathrm{~V} 100 \mathrm{pA})$. All manipulations are performed on selected dark sites (white circles) with a tunneling current of $6 \mathrm{nA}$ for $50 \mathrm{~ms}$ at given bias voltages. (a) and (b) A dark site manipulation at $+9.1 \mathrm{~V}$ shows the dark site displaced to a neighboring adatom. (c) and (d) A dark site manipulation at $+9.8 \mathrm{~V}$ shows the site transformed into a bright site. (e) and (f) A dark site is removed at $+5.0 \mathrm{~V}$.

final possible manipulation outcome for dark sites, in Figs. 5(e) and $5(f)$ the atom returns to normal contrast which represents the desorption of the surface adatom-bonded oxygen.

The three possible manipulations of bright sites are shown in Fig. 6. In Figs. 6(a) and 6(b) the original bright site remains and a new dark site is created on a neighboring atom, described in the literature by the notation $\mathrm{B} \rightarrow \mathrm{B}+\mathrm{D}$. This manipulation can only occur if there are at least two oxygen atoms in back bonds under the original atom. The current injection causes one of them to move to the neighboring adatom, reacting with the dangling bond to create a new dark site, while at least one oxygen remains in the original back bond maintaining the bright site. In Figs. 6(c) and 6(d), a bright site is transformed into a dark site in the same position $(B \rightarrow D)$. This represents an oxygen in a back bond coming to the surface to create a dark site instead. In the last example for bright site manipulation in Figs. 6(e) and 6(f), the bright site is removed (the adatom returns to background contrast) and a new dark site is created near the injection site $\left(\mathrm{B} \rightarrow_{-}+\mathrm{D}\right)$. This represents most likely a single back-bonded oxygen migrating to a neighboring adatom to create the new dark site. 


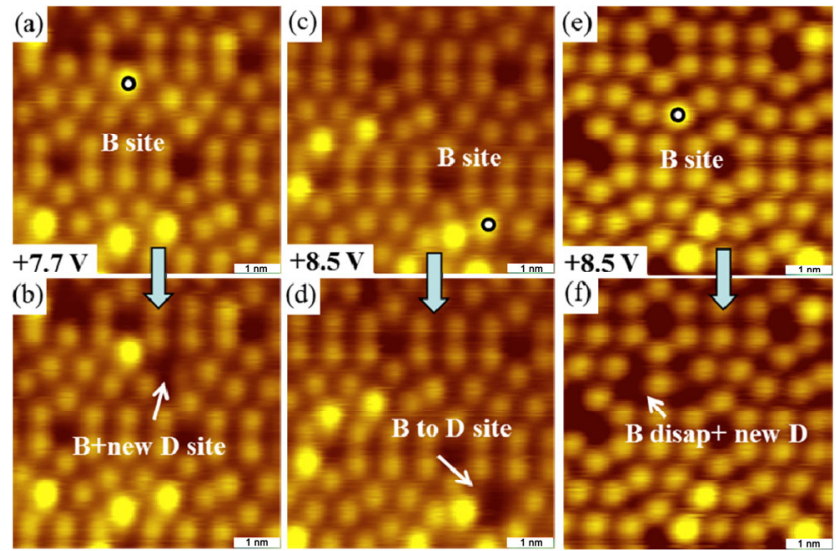

Figure 6 Manipulation of bright sites: before and after $6 \mathrm{~nm} \times 6 \mathrm{~nm}$ STM images taken at the stable imaging bias $(+1.5 \mathrm{~V} 100 \mathrm{pA})$. All manipulations are performed on selected bright sites (white circles) with a tunneling current of $6 \mathrm{nA}$ for $50 \mathrm{~ms}$ at given bias voltages. (a) and (b) At $+7.7 \mathrm{~V}$ the bright site remains and a new dark site appears on a neighboring adatom. (c) and (d) At $+8.5 \mathrm{~V}$ the bright site is transformed into a dark site. (e) and (f) At $+8.5 \mathrm{~V}$ the bright site is removed and a new dark site created.

Thirty-eight bright site injections here at high dose yielded thirteen successful manipulation events which are shown in Table 2 combined with results from twenty-three bright site manipulations at low dose performed by Mayne [29]. At low dose the predominant observed manipulation was for a bright site to transform to a dark site $(B \rightarrow D)$ which represents an oxygen in a back bond coming to the surface to bond with the adatom instead. However, for our higher dose oxygen exposure there is almost a direct flip in the table between the proportion of this $\mathrm{B} \rightarrow \mathrm{D}$ manipulation and the bright site remaining plus a new dark site creation on a neighboring adatom $(B \rightarrow B+D)$. This manipulation can only occur if the injection site has at least two oxygen atoms in back bonds under this original site. The swap in proportion of these two manipulations suggests that at higher oxygen doses more oxygen atoms are inserted into back bonds on bright sites, yielding a large increase in the number of transformations that are characteristic of multiple inserted oxygen atoms, while those that do not require multiple inserted oxygens reduce in turn.

\section{Discussion}

It is now generally accepted by both experimental and density functional theory (DFT) studies that molecular oxygen on $\mathrm{Si}(111)-7 \times 7$ is unstable and spontaneously dissociates upon adsorption [41, 42, 49, 50]. Some DFT studies assume that both of these oxygen atoms must then be incorporated in to the surface [49], however our manipulation studies show that two single-oxygen configurations are possible. Firstly, we find that dark sites can be formed where oxygen bonds only to a surface adatom, with no oxygen inserted into back bonds, usually abbreviated as the ad structure [44]. This ad structure has been considered before in experiment and DFT modeling [42, 44]. While it cannot be detected with STM imaging, we find evidence for it here with local manipulation. Secondly, we are also able

Table 2 Comparison of bright site manipulations via STM current injection for low dose oxygen (Mayne [29]) and higher dose (this work)

\begin{tabular}{ccc}
\hline Manipulation & Low dose (\%) & High dose (\%) \\
\hline $\mathrm{B} \rightarrow \mathrm{D}$ & 43.5 & 23.1 \\
$\mathrm{~B} \rightarrow{ }_{-}+\mathrm{D}$ & 30.4 & 30.8 \\
$\mathrm{~B} \rightarrow \mathrm{B}+\mathrm{D}$ & 26.1 & 46.1 \\
\hline
\end{tabular}

to confirm the singly-inserted oxygen "ins" structure using manipulation. Atomic force microscopy (AFM) can also differentiate between the singly-inserted (ins) and doublyinserted (ins $\times 2$ ) structure from the increase in the upward relaxation of the silicon adatom above [45]. Our oxidation pathway therefore starts from the assumption of spontaneous dissociation of molecular oxygen and initially considers just one oxygen atom reacting with the surface.

At low dose the predominant form is for oxygen to insert in to a back bond, lifting up the relevant adatom and producing a bright site. The absolute number of these inserted-oxygen bright sites increases with dose, but imaging with the STM alone cannot determine how many oxygen atoms have been inserted into back bonds-All sites appear equally brighter. However, by studying the manipulation rates we are able to distinguish between singly-inserted (ins) and at least doubly-inserted oxygen (ins $\times n, n=2,3$ ) sites. We find that although it is possible to have more than one oxygen atom inserted for a bright site even at low dose, the increase in the proportion of $\mathrm{B} \rightarrow \mathrm{B}+\mathrm{D}$ manipulations at high dose, and the corresponding reduction of $\mathrm{B} \rightarrow \mathrm{D}$ manipulations, suggest that predominantly single atom insertion occurs at low dose. This agrees with DFT studies which find that the inserted oxygen structures (ins $\times n, n=1,2,3$ ) are the most stable and form preferentially. Therefore, our oxidation pathway shows the primary singly-inserted ins structure as the first step.

Local manipulations requiring at least two inserted oxygen atoms increase at higher dose while manipulation processes that do not require multiple oxygens reduce, suggesting that the proportion of inserted oxygen atoms increases with dose. These (at least) doubly-inserted bright sites (ins $\times n, n=2,3$ ), shown in the bottom portion of Fig. 3(c), would be indistinguishable in STM imaging at $+1.5 \mathrm{~V}$ from the singly-inserted oxygen bright sites (ins), but behave statistically differently during manipulation via current injection. Both forms of bright site eventually go on to accept an oxygen on the adatom which transforms them to a dark site, which forms the final steps in our pathway.

With the direct creation of ad sites, as well as the conversion of existing bright sites into dark sites (ins $\times n \rightarrow$ ad-ins $\times n$ ), dark site creation overtakes the number of bright sites as dose increases. Further dosing leads eventually to a surface dominated by oxygen-saturated dark sites, followed upon continued oxygen exposure to the creation of thin oxide films on which atomic resolution STM is no longer possible, as observed by us and others [40]. This is also in agreement with DFT studies which find that after inserted structures, the most favorable form energetically is for the combined inserted and adsorbed oxygen forms (ad-ins $\times n$ ) [49].

As well as dose affecting which structure forms, the specific bonding site within the surface reconstruction also changes. Previous STM studies have shown a 2:1 preference for inserted bright sites to bond to the faulted half of the unit cell at low dose compared to the unfaulted half, confirmed by DFT to be energetically preferential $[49,51,52]$. We find the same ratio of 2.0 at $0.3 \mathrm{~L}$, increasing to 2.6 at $0.4 \mathrm{~L}$. We also find a preference of dark sites to form in the faulted half with a ratio to the unfaulted half of 1.6 at $0.3 \mathrm{~L}$ increasing to 2.3 at $0.4 \mathrm{~L}$. DFT studies confirm that the faulted half is also preferential for ad-ins structures, in agreement [49]. In line with DFT and other experimental studies, we find that the inserted bright sites preferentially form at faulted corner hole sites with a ratio of 2.0 for $0.3 \mathrm{~L}$ increasing to 2.3 at $0.4 \mathrm{~L}$ [49]. We find the only exception to the corner hole preference is for dark sites at the higher $0.4 \mathrm{~L}$ dose, where center holes become preferential on 
both the faulted and unfaulted half of the unit cell. Niu et al. found that the ad-ins $\times 3$ structure preferentially forms at center holes on both the faulted and unfaulted halves [49]. The ad-ins $\times 3$ structure, which requires repeated oxidation, would be expected to form more at higher dose. We therefore match experimentally their predication of increasing center hole reactions for this high dose structure.

In summary, STM imaging alone cannot distinguish between singly- and (at least) doubly-inserted oxygen sites, nor can it determine whether a saturated dark site sits on top of inserted oxygen. However, combining STM imaging with local atomic manipulation can reveal the dose-dependent behavior of these sites and inform our understanding of how oxygen reacts with the silicon surface to form the initial oxide.

\section{Acknowledgements}

The authors thank the Engineering and Physical Sciences Research Council (UK) for financial support of this research (No. EP/K006061/2). D. K. thanks the Republic of Turkey Ministry of National Education for a PhD scholarship.

Open Access: This article is licensed under a Creative Commons Attribution 4.0 International License, which permits use, sharing, adaptation, distribution and reproduction in any medium or format, as long as you give appropriate credit to the original author(s) and the source, provide a link to the Creative Commons licence, and indicate if changes were made.

The images or other third party material in this article are included in the article's Creative Commons licence, unless indicated otherwise in a credit line to the material. If material is not included in the article's Creative Commons licence and your intended use is not permitted by statutory regulation or exceeds the permitted use, you will need to obtain permission directly from the copyright holder.

To view a copy of this licence, visit http://creativecommons.org/ licenses/by/4.0/.

\section{References}

[1] Tilli, M.; Motooka, T.; Airaksinen, V. M.; Franssila, A.; PaulastoKröckel, M.; Lindroos, V. Handbook of Silicon Based MEMS Materials and Technologies, 2nd ed.; Amsterdam: William Andrew, 2015.

[2] McNerny, D. Q.; Viswanath, B.; Copic, D.; Laye, F. R.; Prohoda, C.; Brieland-Shoultz, A. C.; Polsen, E. S.; Dee, N. T.; Veerasamy, V. S.; Hart, A. J. Direct fabrication of graphene on $\mathrm{SiO}_{2}$ enabled by thin film stress engineering. Sci. Rep. 2014, 4, 5049.

[3] Zhu, W. H.; Zheng, G.; Cao, S.; He, H. Thermal conductivity of amorphous $\mathrm{SiO}_{2}$ thin film: A molecular dynamics study. Sci. Rep. 2018, 8, 10537.

[4] Wang, Y. M.; Ding, K.; Sun, B. Q.; Lee, S. T.; Jie, J. S. Twodimensional layered material/silicon heterojunctions for energy and optoelectronic applications. Nano Res. 2016, 9, 72-93.

[5] Yu, M.; Xu, W.; Benjalal, Y.; Barattin, R.; Lægsgaard, E.; Stensgaard, I.; Hliwa, M.; Bouju, X.; Gourdon, A.; Joachim, C. et al. STM manipulation of molecular moulds on metal surfaces. Nano Res. 2009, 2, 254-259.

[6] Li, Z.; Schouteden, K.; Iancu, V.; Janssens, E.; Lievens, P.; van Haesendonck, C.; Cerdá, J. I. Chemically modified STM tips for atomic-resolution imaging of ultrathin $\mathrm{NaCl}$ films. Nano Res. 2015, 8, 2223-2230.

[7] Murata, Y.; Cavallucci, T.; Tozzini, V.; Pavliček, N.; Gross, L.; Meyer, G.; Takamura, M.; Hibino, H.; Beltram, F.; Heun, S. Atomic and electronic structure of $\mathrm{Si}$ dangling bonds in quasi-free-standing monolayer graphene. Nano Res. 2018, 11, 864-873.

[8] Krasnikov, S. A.; Lübben, O.; Murphy, B. E.; Bozhko, S. I.; Chaika, A. N.; Sergeeva, N. N.; Bulfin, B.; Shvets, I. V. Writing with atoms: Oxygen adatoms on the $\mathrm{MoO}_{2} / \mathrm{Mo}(110)$ surface. Nano Res. 2013, 6 ,
929-937.

[9] MacLeod, J. M.; Lipton-Duffin, J.; Fu, C. Y.; Rosei, F. Inducing nonlocal reactions with a local probe. ACS Nano 2009, 3, 3347-3351.

[10] Maeda, K.; Nakamura, Y. Spreading effects in surface reactions induced by tunneling current injection from an STM tip. Surf. Sci. 2003, 528, 110-114.

[11] Nakamura, Y.; Mera, Y.; Maeda, K. Nanoscale imaging of electronic surface transport probed by atom movements induced by scanning tunneling microscope current. Phys. Rev. Lett. 2002, 89, 266805.

[12] Lu, X. K.; Polanyi, J. C.; Yang, J. A reversible molecular switch based on pattern-change in chlorobenzene and toluene on a $\mathrm{Si}(111)-(7 \times 7)$ surface. Nano Lett. 2006, 6, 809-814.

[13] Maksymovych, P.; Dougherty, D. B.; Zhu, X. Y.; Yates, J. T., Jr. Nonlocal dissociative chemistry of adsorbed molecules induced by localized electron injection into metal surfaces. Phys. Rev. Lett. 2007, 99, 016101.

[14] Persson, B. N. J.; Avouris, P. Local bond breaking via STM-induced excitations: The role of temperature. Surf. Sci. 1997, 390, 45-54.

[15] Schull, G.; Berndt, R. Orientationally ordered $(7 \times 7)$ superstructure of $\mathrm{C}_{60}$ on $\mathrm{Au}(111)$. Phys. Rev. Lett. 2007, 99, 226105.

[16] Sloan, P. A.; Sakulsermsuk, S.; Palmer, R. E. Nonlocal desorption of chlorobenzene molecules from the $\mathrm{Si}(111)-(7 \times 7)$ surface by charge injection from the tip of a scanning tunneling microscope: Remote control of atomic manipulation. Phys. Rev. Lett. 2010, 105, 048301.

[17] Sakulsermsuk, S.; Sloan, P. A.; Palmer, R. E. A new mechanism of atomic manipulation: Bond-selective molecular dissociation via thermally activated electron attachment. ACS Nano 2010, 4, 7344-7348.

[18] Sloan, P. A.; Palmer, R. E. Two-electron dissociation of single molecules by atomic manipulation at room temperature. Nature $\mathbf{2 0 0 5}, 434,367-371$.

[19] Stipe, B. C.; Rezaei, M. A.; Ho, W. Site-specific displacement of Si adatoms on $\mathrm{Si}(111)-(7 \times 7)$. Phys. Rev. Lett. 1997, 79, 4397-4400.

[20] Inami, E.; Hamada, I.; Ueda, K.; Abe, M.; Morita, S.; Sugimoto, Y. Room-temperature-concerted switch made of a binary atom cluster. Nat. Commun. 2015, 6, 6231.

[21] Ko, E.; Lee, K. R.; Choi, H. J. Tunneling properties versus electronic structures in $\mathrm{Si} / \mathrm{SiO}_{2} / \mathrm{Si} \mathrm{junctions} \mathrm{from} \mathrm{first} \mathrm{principles.} \mathrm{Phys.} \mathrm{Rev.} B$ 2013, 88, 035318.

[22] Medina, H.; Lin, Y. C.; Jin, C. H.; Lu, C. C.; Yeh, C. H.; Huang, K. P.; Suenaga, K.; Robertson, J.; Chiu, P. W. Metal-free growth of nanographene on silicon oxides for transparent conducting applications. Adv. Funct. Mater. 2012, 22, 2123-2128.

[23] Tan, H. R.; Babal, P.; Zeman, M.; Smets, A. H. M. Wide bandgap p-type nanocrystalline silicon oxide as window layer for high performance thin-film silicon multi-junction solar cells. Solar Energy Mater. Solar Cells 2015, 132, 597-605.

[24] Ishizaka, A.; Iwata, $\mathrm{S} . \mathrm{Si}_{-} \mathrm{SiO}_{2}$ interface characterization from angular dependence of X-ray photoelectron spectra. Appl. Phys. Lett. 1980, $36,71-73$.

[25] Muller, D. A.; Sorsch, T.; Moccio, S.; Baumann, F. H.; EvansLutterodt, K.; Timp, G. The electronic structure at the atomic scale of ultrathin gate oxides. Nature 1999, 399, 758-761.

[26] Hollinger, G.; Himpsel, F. J. Probing the transition layer at the $\mathrm{SiO}_{2}-\mathrm{Si}$ interface using core level photoemission. Appl. Phys. Lett. 1984, 44, 93-95.

[27] Oh, J. H.; Yeom, H. W.; Hagimoto, Y.; Ono, K.; Oshima, M.; Hirashita, N.; Nywa, M.; Toriumi, A.; Kakizaki, A. Chemical structure of the ultrathin $\mathrm{SiO}_{2} / \mathrm{Si}(100)$ interface: An angle-resolved $\mathrm{Si} 2 \mathrm{p}$ photoemission study. Phys. Rev. B 2001, 63, 205310.

[28] Höfer, U.; Morgen, P.; Wurth, W.; Umbach, E. Initial stages of oxygen adsorption on $\mathrm{Si}(111)$. II. The molecular precursor. Phys. Rev. B 1989, 40, 1130-1145.

[29] Mayne, A. J.; Rose, F.; Comtet, G.; Hellner, L.; Dujardin, G. Variable temperature STM studies of the adsorption of oxygen on the Si(111)-7×7 surface. Surf. Sci. 2003, 528, 132-137.

[30] Martel, R.; Avouris, P.; Lyo, I. W. Molecularly adsorbed oxygen species on $\operatorname{Si}(111)-(7 \times 7)$ : STM-induced dissociative attachment studies. Science 1996, 272, 385-388.

[31] Rusimova, K. R.; Purkiss, R. M.; Howes, R.; Lee, F.; Crampin, S.; Sloan, P. A. Regulating the femtosecond excited-state lifetime of a single molecule. Science 2018, 361, 1012-1016. 
[32] Rusimova, K. R.; Bannister, N.; Harrison, P.; Lock, D.; Crampin, S.; Palmer, R. E.; Sloan, P. A. Initiating and imaging the coherent surface dynamics of charge carriers in real space. Nat. Commun. 2016, 7 , 12839.

[33] Rusimova, K. R.; Sloan, P. A. Molecular and atomic manipulation mediated by electronic excitation of the underlying $\operatorname{Si}(111)-7 \times 7$ surface. Nanotechnology 2016, 28, 054002.

[34] Lock, D.; Rusimova, K. R.; Pan, T. L.; Palmer, R. E.; Sloan, P. A. Atomically resolved real-space imaging of hot electron dynamics. Nat. Commun. 2015, 6, 8365.

[35] Lock, D.; Sakulsermsuk, S.; Palmer, R. E.; Sloan, P. A. Mapping the site-specific potential energy landscape for chemisorbed and physisorbed aromatic molecules on the $\operatorname{Si}\left(\begin{array}{llll}1 & 1 & 1\end{array}\right)-7 \times 7$ surface by time-lapse STM. J. Phys. Condens. Matter 2015, 27, 054003.

[36] Pan, T. L.; Sloan, P. A.; Palmer, R. E. Concerted thermal-plus-electronic nonlocal desorption of chlorobenzene from $\mathrm{Si}(111)-7 \times 7$ in the STM. J. Phys. Chem. Lett. 2014, 5, 3551-3554.

[37] Pan, T. L.; Sloan, P. A.; Palmer, R. E. Non-local atomic manipulation on semiconductor surfaces in the STM: The case of chlorobenzene on $\mathrm{Si}(111)-7 \times 7$. Chem. Rec. 2014, 14, 841-847.

[38] Sakulsermsuk, S.; Sloan, P. A.; Theis, W.; Palmer, R. E. Calibrating thermal and scanning tunnelling microscope induced desorption and diffusion for the chemisorbed chlorobenzene/Si(111)7×7 system. $J$. Phys. Condens. Matter 2010, 22, 084002.

[39] Sloan, P. A.; Hedouin, M. F. G.; Palmer, R. E.; Persson, M. Mechanisms of molecular manipulation with the scanning tunneling microscope at room temperature: Chlorobenzene/Si(111)-(7×7). Phys. Rev. Lett. 2003, 91,118301

[40] Lyo, I. W.; Avouris, P. Field-induced nanometer- to atomic-scale manipulation of silicon surfaces with the STM. Science 1991, 253, 173-176.

[41] Lee, S. H.; Kang, M. H. Origin of O 1s core-level shifts on oxygen adsorbed $\mathrm{Si}(111)-(7 \times 7)$. Phys. Rev. Lett. 2000, 84, 1724-1727.

[42] Lee, S. H.; Kang, M. H. Identification of the initial-stage oxidation products on $\mathrm{Si}(111)-(7 \times 7)$. Phys. Rev. Lett. 1999, 82, 968-971.

[43] Comtet, G.; Hellner, L.; Dujardin, G.; Bobrov, K. Adsorption of $\mathrm{O}_{2}$ on $\mathrm{Si}(111)-7 \times 7$ at 300 and $30 \mathrm{~K}$ studied by ion photodesorption and electron photoemission. Phys. Rev. B 2001, 65, 035315.

[44] Sakamoto, K.; Doi, S.; Ushimi, Y.; Ohno, K.; Yeom, H. W.; Ohta, T.; Suto, S.; Uchida, W. Adsorption process of metastable molecular oxygen on a Si(111)-7×7 surface. Phys. Rev. B 1999, 60, R8465-R8468.

[45] Onoda, J.; Ondráček, M.; Yurtsever, A.; Jelínek, P.; Sugimoto, Y. Initial and secondary oxidation products on the $\mathrm{Si}(111)-(7 \times 7)$ surface identified by atomic force microscopy and first principles calculations. Appl. Phys. Lett. 2014, 104, 133107.

[46] Leibsle, F. M.; Samsavar, A.; Chiang, T. C. Oxidation of Si(111)-(7×7) as studied by scanning tunneling microscopy. Phys. Rev. B 1988, 38, 5780-5783.

[47] Tokumoto, H.; Miki, K.; Murakami, H.; Bando, H.; Ono, M.; Kajimura, K. Real-time observation of oxygen and hydrogen adsorption on silicon surfaces by scanning tunneling microscopy. $J$. Vac. Sci. Technol. A 1990, 8, 255-258.

[48] Kaya, D. The effect of electric field on a fullerene molecule on a metal surface by a nano STM tip. Phys. B Condens. Matter 2019, 557, 126-131.

[49] Niu, C. Y.; Wang, J. T. Adsorption and dissociation of oxygen molecules on Si(111)-(7×7) surface. J. Chem. Phys. 2013, 139, 194709.

[50] Wiggins, B.; Avila-Bront, L. G.; Edel, R.; Sibener, S. J. Temporally and spatially resolved oxidation of $\mathrm{Si}(111)-(7 \times 7)$ using kinetic energy controlled supersonic beams in combination with scanning tunneling microscopy. J. Phys. Chem. C 2016, 120, 8191-8197.

[51] Hasegawa, T.; Kohno, M.; Hosoki, S. Initial stage of oxygen adsorption onto a $\mathrm{Si}(111)-7 \times 7$ surface studied by scanning tunneling microscopy. Jpn. J. Appl. Phys. 1994, 33, 3702-3705.

[52] Caldas, M. J.; Baierle, R. J.; Capaz, R. B.; Artacho, E. Ab initio study of atomic oxygen adsorption on the $\mathrm{Si}(111) 7 \times 7$ surface. Phys. B Condens. Matter 2001, 308-310, 329-332. 